\title{
Fourth-Order Compact Schemes of a Heat Conduction Problem with Neumann Boundary Conditions
}

\author{
Jennifer Zhao, ${ }^{1}$ Weizhong Dai, ${ }^{2}$ Tianchan Niu ${ }^{2}$ \\ ${ }^{1}$ Department of Mathematics and Statistics, University of Michigan-Dearborn, \\ Dearborn, MI 48128 \\ ${ }^{2}$ Program of Mathematics and Statistics, College of Engineering and Science, \\ Louisiana Tech University, Ruston, LA 71272
}

Received 10 May 2006; accepted 10 August 2006

Published online 18 May 2007 in Wiley InterScience (www.interscience.wiley.com).

DOI 10.1002/num. 20200

\begin{abstract}
In this article, a set of fourth-order compact finite difference schemes is developed to solve a heat conduction problem with Neumann boundary conditions. It is derived through the compact difference schemes at all interior points, and the combined compact difference schemes at the boundary points. This set of schemes is proved to be globally solvable and unconditionally stable. Numerical examples are provided to verify the accuracy. (c) 2007 Wiley Periodicals, Inc. Numer Methods Partial Differential Eq 23: 949-959, 2007
\end{abstract}

Keywords: compact finite difference schemes; Neumann boundary conditions; heat conduction problem

\section{INTRODUCTION}

High-order compact schemes (HOCs) are frequently used because they can provide accurate results on well-structured compact stencils. Most existing high-order compact schemes are constructed for problems with Dirichlet boundary conditions (see [1-12] for references). However, fewer HOCs have been constructed for problems with Neumann boundary conditions, which are much more difficult to handle than Dirichlet conditions. Even for those less popular compact difference schemes involving Neumann boundary conditions, very often, the schemes are fourth or sixth order at the interior points, but only second order or less at the boundary points [3].

In this article, we construct a set of fourth-order compact finite difference schemes for a heat conduction problem with Neumann boundary conditions. Rather than being second order or less at the boundary points, the new set of schemes is fourth-order accurate in space at all grid points, both interior and boundary points. Moreover, it is proved to be globally solvable and unconditionally stable. It should be pointed out that the method used in proving the stability result in this

Correspondence to: Jennifer Zhao, Department of Mathematics and Statistics, University of Michigan-Dearborn, 4901 Evergreen Road, Dearborn, MI 48128 (e-mail: xich@umich.edu)

AMS subject classification: 65M06, 65M12

(C) 2007 Wiley Periodicals, Inc. 
article is quite different from the traditional approaches, because we use both matrix analysis and the quadratic forms of analysis.

The set of schemes is constructed based on the following heat conduction problem:

$$
\begin{aligned}
& U_{t}=\beta U_{x x}+F(x, t), \quad 0<x<1, \quad t>0, \\
& U(x, 0)=U_{0}(x), \\
& U_{x}(0, t)=\alpha_{1}(t), \quad U_{x}(1, t)=\alpha_{2}(t) .
\end{aligned}
$$

Here $U(x, t)$ represents the temperature at point $(x, t) ; \beta$ is the diffusion constant; and $F(x, t)$, $\alpha_{1}(t)$, and $\alpha_{2}(t)$ are sufficiently smooth functions.

The outline of this article is as follows. In Section 2, the set of fourth-order compact difference schemes is constructed for both interior and boundary points. Its global solvability and unconditional stability results are stated and proved in Section 3. Numerical examples are provided in Section 4.

\section{THE SET OF SCHEMES}

\subsection{Preliminaries}

Let $\Delta x$ and $\Delta t$ denote the spatial mesh size and time increment, respectively. We assume that there exists an integer $M$, such that $(M+1) \Delta x=1$, and $u_{j}^{m}$ and $\left(u_{x x}\right)_{j}^{m}$ are used to represent the numerical approximations of $U(j \Delta x, m \Delta t)$ and $U_{x x}(j \Delta x, m \Delta t)$, respectively. Also, we use $j$ and $m$ to represent the discrete space and time indexes, where $0 \leq j \leq M+1$ and $m \geq 0$.

The application of the well-known Crank-Nicolson type of scheme to (1) results in the following expression:

$$
\frac{u_{j}^{m+1}-u_{j}^{m}}{\Delta t}=\frac{\left(u_{x x}\right)_{j}^{m+1}+\left(u_{x x}\right)_{j}^{m}}{2}+F_{j}^{m+1 / 2},
$$

where

$$
F_{j}^{m+1 / 2}=\frac{F\left(x_{j}, t^{m}\right)+F\left(x_{j}, t^{m+1}\right)}{2} .
$$

Based on the established results of the Crank-Nicolson type of scheme, (2) is order $O\left(\Delta t^{2}\right)$ in time. In the next two subsections, we will construct fourth-order approximations to terms $\left(u_{x x}\right)_{j}^{m}$ and $\left(u_{x x}\right)_{j}^{m+1}$, so that (2) is order $O\left(\Delta x^{4}\right)$ in space.

\subsection{The Interior Points}

The first part of our construction is for all interior points $\left(x_{j}, t^{m}\right)$, where $2 \leq j \leq M-1$ and $m \geq 1$.

The following compact scheme is used in order to derive a fourth-order approximation to $\left(u_{x x}\right)_{j}^{m}$ :

$$
\begin{aligned}
& \frac{U\left(x_{j+1}, t^{m}\right)-2 U\left(x_{j}, t^{m}\right)+U\left(x_{j-1}, t^{m}\right)}{\Delta x^{2}} \\
& \quad=a U_{x x}\left(x_{j+1}, t^{m}\right)+b U_{x x}\left(x_{j}, t^{m}\right)+c U_{x x}\left(x_{j-1}, t^{m}\right),
\end{aligned}
$$


where $a, b$, and $c$ are constants to be determined. The Taylor series expansions to terms $U\left(x_{j+1}, t^{m}\right)$ and $U\left(x_{j-1}, t^{m}\right)$, on the left side of (3), at the point $\left(x_{j}, t^{m}\right)$ yield the following result:

$$
\frac{U\left(x_{j+1}, t^{m}\right)-2 U\left(x_{j}, t^{m}\right)+U\left(x_{j-1}, t^{m}\right)}{\Delta x^{2}}=U_{x x}\left(x_{j}, t^{m}\right)+\frac{\Delta x^{2}}{12} U_{x x x x}\left(x_{j}, t^{m}\right)+O\left(\Delta x^{4}\right) .
$$

Similar expansions for $U_{x x}\left(x_{j+1}, t^{m}\right)$ and $U_{x x}\left(x_{j-1}, t^{m}\right)$, on the right side of (3), at the same point yield the result below:

$$
\begin{gathered}
U_{x x}\left(x_{j \pm 1}, t^{m}\right)=U_{x x}\left(x_{j}, t^{m}\right) \pm \Delta x U_{x x x}\left(x_{j}, t^{m}\right)+\frac{\Delta x^{2}}{2} U_{x x x x}\left(x_{j}, t^{m}\right) \\
\pm \frac{\Delta x^{3}}{3 !} U_{x x x x x}\left(x_{j}, t^{m}\right)+O\left(\Delta x^{4}\right) .
\end{gathered}
$$

The substitutions of (3) into (4) and (5) give the next result:

$$
\begin{aligned}
& U_{x x}\left(x_{j}, t^{m}\right)+\frac{\Delta x^{4}}{12} U_{x x x x}\left(x_{j}, t^{m}\right)+O\left(\Delta x^{4}\right) \\
& =(a+b+c) U_{x x}\left(x_{j}, t^{m}\right)+(-a+c) \Delta x U_{x x x}\left(x_{j}, t^{m}\right) \\
& \quad+\frac{a+c}{2} \Delta x^{2} U_{x x x x}\left(x_{j}, t^{m}\right)+\frac{-a+c}{6} \Delta x^{3} U_{x x x x x}\left(x_{j}, t^{m}\right)+O\left(\Delta x^{4}\right) .
\end{aligned}
$$

To turn both sides of (6) into fourth order, we need to equate the corresponding coefficients for terms involving $\Delta x^{0}, \Delta x, \Delta x^{2}$, and $\Delta x^{3}$ on both sides. This results in the following set of linear equations in terms of $a, b$, and $c$ :

$$
a+b+c=1, \quad a-c=0, \quad \frac{a+c}{2}=\frac{1}{12}, \quad \frac{a-c}{6}=0,
$$

for which the solutions are

$$
a=c=\frac{1}{12}, \quad b=\frac{5}{6} .
$$

Substituting these values into (3) and changing $U\left(x_{j-1}, t^{m}\right), U\left(x_{j}, t^{m}\right), U\left(x_{j+1}, t^{m}\right)$ and $U_{x x}\left(x_{j-1}, t^{m}\right), U_{x x}\left(x_{j}, t^{m}\right)$ and $U_{x x}\left(x_{j+1}, t^{m}\right)$ to their analogous notations, the following fourthorder relation at all interior points and any time level, is derived.

$$
\begin{aligned}
& \frac{1}{10}\left(u_{x x}\right)_{j-1}^{m}+\left(u_{x x}\right)_{j}^{m}+\frac{1}{10}\left(u_{x x}\right)_{j+1}^{m}=\frac{6}{5 \Delta x^{2}}\left(u_{j-1}^{m}-2 u_{j}^{m}+u_{j+1}^{m}\right), \\
& 2 \leq j \leq M-1, \quad m \geq 1 .
\end{aligned}
$$

It should be pointed out that (7) is as same as that in [4]. Furthermore, it can be seen that (7) is obtained by solving a linear system, which is different from the standard approximations such as the following one, which is obtained from Taylor series only:

$$
\left(u_{x x}\right)_{j}=\frac{u_{j+1}-2 u_{j}+u_{j-1}}{\Delta x^{2}}+O\left(\Delta x^{2}\right)
$$




\subsection{The Boundary Points}

We now develop similar fourth-order approximations for $\left(u_{x x}\right)\left(x_{1}, t^{m}\right)$ and $\left(u_{x x}\right)\left(x_{M}, t^{m}\right)$, where $x_{1}$ and $x_{M}$ are the two points next to the actual boundary points 0 and 1 . We start with $\left(x_{1}, t^{m}\right)$ first, where the following combined compact scheme is used:

$$
\frac{b^{*}}{\Delta x} U_{x}\left(0, t^{m}\right)+a^{*} U_{x x}\left(x_{1}, t^{m}\right)+c^{*} U_{x x}\left(x_{2}, t^{m}\right)=\frac{1}{\Delta x^{2}}\left(e^{*} U\left(x_{1}, t^{m}\right)+f^{*} U\left(x_{2}, t^{m}\right)\right),
$$

$a^{*}, b^{*}, c^{*}, e^{*}$, and $f^{*}$ are constants to be determined. By multiplying both sides of (9) by $\Delta x^{2}$, we can rewrite it as follows:

$$
b^{*} \Delta x U_{x}\left(0, t^{m}\right)+a^{*} \Delta x^{2} U_{x x}\left(x_{1}, t^{m}\right)+c^{*} \Delta x^{2} U_{x x}\left(x_{2}, t^{m}\right)=e^{*} U\left(x_{1}, t^{m}\right)+f^{*} U\left(x_{2}, t^{m}\right) .
$$

If each term of (10) is expanded in Taylor series at point $\left(0, t^{m}\right)$, we would obtain the following results:

$$
\begin{gathered}
U\left(x_{1}, t^{m}\right)=U\left(0, t^{m}\right)+\Delta x U_{x}\left(0, t^{m}\right)+\frac{\Delta x^{2}}{2} U_{x x}\left(0, t^{m}\right)+\frac{\Delta x^{3}}{6} U_{x x x}\left(0, t^{m}\right)+O\left(\Delta x^{4}\right) . \\
U\left(x_{2}, t^{m}\right)=U\left(0, t^{m}\right)+2 \Delta x U_{x}\left(0, t^{m}\right)+\frac{(2 \Delta x)^{2}}{2 !} U_{x x}\left(0, t^{m}\right) \\
+\frac{(2 \Delta x)^{3}}{3 !} U_{x x x}\left(0, t^{m}\right)+O\left(\Delta x^{4}\right) . \\
U_{x x}\left(x_{1}, t^{m}\right)=U_{x x}\left(0, t^{m}\right)+\Delta x U_{x x x}\left(0, t^{m}\right)+O\left(\Delta x^{2}\right) . \\
U_{x x}\left(x_{2}, t^{m}\right)=U_{x x}\left(0, t^{m}\right)+2 \Delta x U_{x x x}\left(0, t^{m}\right)+O\left(\Delta x^{2}\right) .
\end{gathered}
$$

The substitutions into (10) of (11-14), and afterward simplifications give the following result:

$$
\begin{aligned}
b^{*} \Delta x & U_{x}\left(0, t^{m}\right)+\left(a^{*}+c^{*}\right) \Delta x^{2} U_{x x}\left(0, t^{m}\right)+\left(a^{*}+2 c^{*}\right) U_{x x x}\left(0, t^{m}\right)+O\left(\Delta x^{4}\right) \\
= & \left(e^{*}+f^{*}\right) U\left(0, t^{m}\right)+\left(e^{*}+2 f^{*}\right) \Delta x U_{x}\left(0, t^{m}\right) \\
& +\left(e^{*}+2 f^{*}\right) \Delta x U_{x x}\left(0, t^{m}\right)+\frac{e^{*}+8 f^{*}}{6} \Delta x^{3} U_{x x x}\left(0, t^{m}\right)+O\left(\Delta x^{4}\right) .
\end{aligned}
$$

To derive a fourth-order approximation in (15), we only need to equate the corresponding coefficients for those terms involving $\Delta x^{0}, \Delta x, \Delta x^{2}$ and $\Delta x^{3}$ on its both sides. This results in the following system equations:

$$
e^{*}+f^{*}=0, \quad b^{*}=e^{*}+2 f^{*}, \quad a^{*}+c^{*}=\frac{e^{*}}{2}+2 f^{*}, \quad a^{*}+2 c^{*}=\frac{e^{*}+8 f^{*}}{6},
$$

with the solutions to be

$$
a^{*}=\frac{11}{6} f^{*}, \quad b^{*}=f^{*}, \quad c^{*}=-\frac{1}{3} f^{*}, \quad e^{*}=-f^{*} .
$$

We take $f^{*}=1$ in the above solutions, and substitute it together with the corresponding values of $a^{*}, b^{*}, c^{*}, d^{*}, e^{*}$ into (10) to get the following scheme:

$$
\frac{11}{6} U_{x x}\left(x_{1}, t^{m}\right)-\frac{1}{3} U_{x x}\left(x_{2}, t^{m}\right)=-\frac{1}{\Delta x} U_{x}\left(0, t^{m}\right)+\frac{U\left(x_{2}, t^{m}\right)-U\left(x_{1}, t^{m}\right)}{\Delta x^{2}} .
$$


After replacing $U\left(x_{j}, t^{m}\right)$ to $u_{j}^{m}$ and applying the boundary condition $U_{x}\left(0, t^{m}\right)=\alpha_{1}\left(t^{m}\right)$, the following fourth-order scheme at the boundary point $\left(x_{1}, t^{m}\right)$ is obtained:

$$
\frac{11}{6}\left(u_{x x}\right)_{1}^{m}-\frac{1}{3}\left(u_{x x}\right)_{2}^{m}=-\frac{\alpha_{1}\left(t^{m}\right)}{\Delta x}+\frac{u_{2}^{m}-u_{1}^{m}}{\Delta x^{2}} .
$$

Similarly, the following fourth-order scheme at the point $\left(x_{M-1}, t^{m}\right)$ can also be derived:

$$
\frac{11}{6}\left(u_{x x}\right)_{M}^{m}-\frac{1}{3}\left(u_{x x}\right)_{M-1}^{m}=\frac{\alpha_{2}\left(t^{m}\right)}{\Delta x}+\frac{u_{M-1}^{m}-u_{M}^{m}}{\Delta x^{2}} .
$$

Thus the set of schemes, consisting of (2), (7), (19), and (20), has overall order of $O\left(\Delta t^{2}+\Delta x^{4}\right)$, which indicates that our set of compact schemes is consistent with the differential equation.

\section{SOLVABILITY AND STABILITY}

\subsection{Solvability}

3.1.1. Matrix Form To prove the solvability and stability results of the set of schemes obtained in the previous section, we first introduce the following vectors at any time level $t^{m}(m \geq 0)$ :

$$
\begin{aligned}
\vec{u}^{m} & =\left(u_{1}^{m}, \ldots, u_{M}^{m}\right)^{T}, \quad\left(\vec{u}_{x x}\right)^{m}=\left(\left(u_{x x}\right)_{1}^{m}, \ldots,\left(u_{x x}\right)_{M}^{m}\right)^{T} \\
\vec{\alpha}\left(t^{m}\right) & =\left(-\frac{\alpha_{1}\left(t^{m}\right)}{\Delta x}, 0,0, \ldots, 0,-\frac{\alpha_{2}\left(t^{m}\right)}{\Delta x}\right)^{T},
\end{aligned}
$$

and the following two square matrices:

$$
A=\left[\begin{array}{rrrrr}
22 & -4 & & & \\
1 & 10 & 1 & & \\
& \ddots & \ddots & \ddots & \\
& & 1 & 10 & 1 \\
& & & -4 & 22
\end{array}\right]_{M \times M} \quad B=\left[\begin{array}{rrrrr}
6 & -6 & & & \\
-6 & 12 & -6 & & \\
& \ddots & \ddots & \ddots & \\
& & -6 & 12 & -6 \\
& & & -6 & 6
\end{array}\right]_{M \times M}
$$

With this notation, the schemes (7), (19), and (20) can be combined and expressed in the following matrix form:

$$
\frac{1}{10} A\left(\vec{u}_{x x}\right)^{m}=-\frac{1}{5 \Delta x^{2}} B \vec{u}^{m}+\frac{6}{5} \vec{\alpha}\left(t^{m}\right) .
$$

By the Gerschgorin Theorem [13], we can prove that $A$ is invertible. This invertibility result also proves the solvability of (23), which can be simplified to:

$$
\left(\vec{u}_{x x}\right)^{m}=-\frac{2}{\Delta x^{2}} A^{-1} B \vec{u}^{m}+12 A^{-1} \vec{\alpha}\left(t^{m}\right) .
$$

3.1.2. Advance from Level $\boldsymbol{t}^{\boldsymbol{n}}$ to Level $\boldsymbol{t}^{\boldsymbol{n}+\boldsymbol{1}}$ Assume that the sets of values $\left\{u_{j}^{m}\right\}$ have been obtained for $0 \leq m \leq n$, where $t^{n}$ represents a fixed time level. We now show that the set of values $\left\{u_{j}^{n+1}\right\}$ can be obtained from $\left\{u_{j}^{n}\right\}$. After the substitutions of (24) into (2), we have the following vector equation:

$$
\frac{\vec{u}^{n+1}-\vec{u}^{n}}{\Delta t}=-\frac{1}{\Delta x^{2}} A^{-1} B\left(\vec{u}^{n+1}+\vec{u}^{n}\right)+6 A^{-1}\left(\vec{\alpha}\left(t^{n+1}\right)+\vec{\alpha}\left(t^{n}\right)\right)+\vec{F}^{n+1 / 2},
$$


where

$$
\vec{F}^{n+1 / 2}=\left(F_{1}^{n+1 / 2}, \ldots, F_{M}^{n+1 / 2}\right)
$$

Equation (25) can be further simplified to the next result:

$$
(A+r B) \vec{u}^{n+1}=(A-r B) \vec{u}^{n}+6 \Delta t B\left(\alpha\left(t^{n+1}\right)+\alpha\left(t^{n}\right)\right)+\Delta t A \vec{F}^{n+1 / 2},
$$

where $r=\Delta t / \Delta x^{2}$. We can prove quite easily that the matrix $A+r B$ is invertible by the Gerschgorin Theorem, the detail is omitted here. It follows that (26) is solvable, therefore, $\left\{u_{j}^{n+1}\right\}$ can be obtained from $\left\{u_{j}^{n}\right\}$. This proves that the set of schemes, consisting of (2), (7), (19), and (20), is globally solvable.

\subsection{Stability}

The stability result is proved in this sub-section. Based on the theory for the finite difference method [14], a consistent and stable finite difference scheme implies its convergence to the exact solution. Hence, only the stability result is proved here, the convergence result follows automatically.

The result and proof are based on (26), which is the vector form of set consisting of (2), (7), (19), and (20). Assume that $\vec{u}_{1}^{m}$ and $\vec{u}_{2}^{m}$ are two different solutions of (26) having two different initial data sets but same boundary conditions. Let $\vec{\theta}^{m}=\vec{u}_{1}^{m}-\vec{u}_{2}^{m}$, then it satisfies the following equation:

$$
\frac{\vec{\theta}^{n+1}-\vec{\theta}^{n}}{\Delta t}=-\frac{1}{\Delta x^{2}} A^{-1} B\left(\vec{\theta}^{n+1}+\vec{\theta}^{n}\right)
$$

at any two consecutive time levels $t^{n}$ and $t^{n+1}$. The following result then follows from (27):

$$
\begin{aligned}
\vec{\theta}^{n+1} & =\left(I+r A^{-1} B\right)^{-1}\left(I-r A^{-1} B\right) \vec{\theta}^{n} \\
& =\left[\left(I+r A^{-1} B\right)^{-1}\left(I-r A^{-1} B\right)\right]^{2} \vec{\theta}^{n-1} \\
& =\cdots \\
& =\left[\left(I+r A^{-1} B\right)^{-1}\left(I-r A^{-1} B\right)\right]^{n+1} \vec{\theta}^{0},
\end{aligned}
$$

where $r=\Delta t / \Delta x^{2}$.

We now prove that the magnitude of each eigenvalue of matrix

$$
\left(I+r A^{-1} B\right)^{-1}\left(I-r A^{-1} B\right)
$$

is real and less than or equal to 1 .

Note that if $\lambda$ is an eigenvalue of $A^{-1} B$, then $(1-r \lambda) /(1+r \lambda)$ is an eigenvalue of the matrix (29) having the same corresponding eigenvectors. Thus, in order to prove that $|1-r \lambda / 1+r \lambda| \leq 1$, we only need to show that each $\lambda$ is real and $\lambda \geq 0$. This proof is provided next.

Lemma 1. Assume that $\lambda$ is an eigenvalue of matrix $A^{-1} B$, and $\vec{x}$, a vector of dimension $M$, is a corresponding eigenvector. Then $\lambda$ is real and satisfies

$$
\lambda \geq 0
$$


Proof. Since $\lambda$ and $\vec{x}$ are an eigenvalue and a corresponding eigenvector of matrix $A^{-1} B$, they satisfy the following conditions:

$$
\lambda \vec{x}=A^{-1} B \vec{x}, \quad \text { or } \quad \lambda \vec{x}^{T} A \vec{x}=\vec{x}^{T} B \vec{x} .
$$

Since

$$
\begin{aligned}
& \vec{x}^{T} A \vec{x}=\left[x_{1}, x_{2}, \cdots, x_{M}\right]\left[\begin{array}{rrrrr}
22 & -4 & & & \\
1 & 10 & 1 & & \\
& \ddots & \ddots & \ddots & \\
& & 1 & 10 & 1 \\
& & & -4 & 22
\end{array}\right]\left[\begin{array}{c}
x_{1} \\
x_{2} \\
\vdots \\
x_{M}
\end{array}\right] \\
& =22 x_{1}^{2}-4 x_{1} x_{2}+x_{1} x_{2}+10 x_{2}^{2}+2 x_{2} x_{3}+10 x_{3}^{3}+2 x_{3} x_{4}+\cdots \\
& +2 x_{M-2} x_{M-1}+10 x_{M-1}^{2}-4 x_{M-1} x_{M}+x_{M-1} x_{M}+22 x_{M}^{2} \\
& \geq 22 x_{1}^{2}-\frac{3}{2}\left(x_{1}^{2}+x_{2}^{2}\right)+10 x_{2}^{2}-\left(x_{2}^{2}+x_{3}^{2}\right)+10 x_{3}^{2}-\left(x_{3}^{2}+x_{4}^{2}\right)+\cdots \\
& +\left(x_{M-2}^{2}+x_{M-1}^{2}\right)+10 x_{M-1}^{2}-\frac{3}{2}\left(x_{M-1}^{2}+x_{M}^{2}\right)+22 x_{M}^{2} \\
& =\frac{41}{2} x_{1}^{2}+\frac{15}{2} x_{2}^{2}+8 x_{3}^{2}+\cdots+8 x_{M-2}^{2}+\frac{15}{2} x_{M-1}^{2}+\frac{41}{2} x_{M}^{2} \\
& >0
\end{aligned}
$$

and

$$
\begin{aligned}
& \vec{x}^{T} B \vec{x}=\left[x_{1}, x_{2}, \ldots, x_{M}\right]\left[\begin{array}{rrrrr}
6 & -6 & & & \\
-6 & 12 & -6 & & \\
& \ddots & \ddots & \ddots & \\
& & -6 & 12 & -6 \\
& & & -6 & 6
\end{array}\right]\left[\begin{array}{c}
x_{1} \\
x_{2} \\
\vdots \\
x_{M}
\end{array}\right] \\
& =6 x_{1}^{2}-6 x_{1} x_{2}-6 x_{1} x_{2}+12 x_{2}^{2}-12 x_{2} x_{3}+12 x_{3}^{2}-12 x_{3} x_{4}-\cdots \\
& -12 x_{M-2} x_{M-1}+12 x_{M-1}^{2}-6 x_{M-1} x_{M}-6 x_{M-1} x_{M}+6 x_{M}^{2} \\
& \geq 6 x_{1}^{2}-6\left(x_{1}^{2}+x_{2}^{2}\right)+12 x_{2}^{2}-6\left(x_{2}^{2}+x_{3}^{2}\right)+12 x_{3}^{2}-6\left(x_{3}^{2}+x_{4}^{2}\right)-\cdots \\
& -6\left(x_{M-2}^{2}+x_{M-1}^{2}\right)+12 x_{M-1}^{2}-6\left(x_{M-1}^{2}+x_{M}^{2}\right)+6 x_{M}^{2} \\
& =0 \text {, }
\end{aligned}
$$

the above two results indicate that $\lambda$ is real and $\lambda \geq 0$.

The stability proof also requires the following lemma [13].

Lemma 2. Let $A$ be an arbitrary square matrix. Then for any operator matrix norm $\|\cdot\|$, we have $\lambda_{\sigma}(A) \leq\|A\|$, here $\lambda_{\sigma}(A)$ represents the spectral radius of $A$. Moreover, if $\epsilon>0$, then there exists an operator matrix norm, denoted here by $\|\cdot\|_{\epsilon}$, such that $\|A\|_{\epsilon} \leq \lambda_{\sigma}(A)+\epsilon$.

Based on this lemma, the stability result of (25) can now be stated as follows. 
Theorem 1. The set of schemes, consisting of (2), (7), (19), and (20) or their equivalent vector form (26), is unconditionally stable with respect to the initial data.

Proof. Lemma 1, (31) and (32) indicate the following result:

$$
\begin{aligned}
& \left\|\left(I+r A^{-1} B\right)^{-1}\left(I-r A^{-1} B\right)\right\|_{\epsilon} \\
& \quad \leq \lambda_{\sigma}\left(\left(I+r A^{-1} B\right)^{-1}\left(I-r A^{-1} B\right)\right)+\epsilon \leq 1+\epsilon,
\end{aligned}
$$

where $\epsilon>0$. From this, it can be concluded that

$$
\begin{aligned}
\left\|\vec{\theta}^{n}\right\|_{\epsilon} & \leq\left\|\left[\left(I+r A^{-1} B\right)^{-1}\left(I-r A^{-1} B\right)\right]^{n}\right\|_{\epsilon}\left\|\vec{\theta}^{0}\right\|_{\epsilon} \\
& \leq\left(\left\|\left(I+r A^{-1} B\right)^{-1}\left(I-r A^{-1} B\right)\right\|_{\epsilon}\right)^{n}\left\|\vec{\theta}^{0}\right\|_{\epsilon} \\
& \leq(1+\epsilon)^{n}\left\|\vec{\theta}^{0}\right\|_{\epsilon} \\
& \leq e^{n \epsilon}\left\|\vec{\theta}^{0}\right\|_{\epsilon} .
\end{aligned}
$$

If we take $\epsilon=\Delta t$, then (34) yields the following result:

$$
\left\|\vec{\theta}^{n}\right\|_{\Delta t} \leq e^{n \Delta t}\left\|\vec{\theta}^{0}\right\|_{\Delta t} \leq e^{T}\left\|\vec{\theta}^{0}\right\|_{\Delta t}
$$

for $t^{n} \leq T$. This marks the end of our proof.

\section{NUMERICAL EXAMPLES}

In this section, numerical examples are provided to verify the accuracy. The new set of schemes obtained in this article is compared to the following standard Crank-Nicolson type of scheme:

$$
\begin{aligned}
\frac{u_{j}^{n+1}-u_{j}^{n}}{\Delta t} & =\delta_{x}^{2}\left(\frac{u_{j}^{n+1}+u_{j}^{n}}{2}\right)+F_{j}^{n+1 / 2}, & & 1 \leq j \leq M, n \geq 0, \\
\frac{u_{1}^{n}-u_{0}^{n}}{\Delta x} & =\alpha_{1}\left(t^{n}\right), \frac{u_{M+1}^{n}-u_{M}^{n}}{\Delta x}=\alpha_{2}\left(t^{n}\right) & & n \geq 0 .
\end{aligned}
$$

The comparison is based on the two following examples described below, where $\beta$ is taken to be 1 .

The computations are performed using $\Delta t=0.0001, \Delta x=0.1,0.02$ and 0.04 for both examples, and the following stopping criterion is applied:

$$
\max _{1 \leq j \leq M}\left|\left(u_{j}^{n+1}\right)^{\text {new }}-\left(u_{j}^{n+1}\right)^{\text {old }}\right|<10^{-8} .
$$

It should be pointed out, although a small $\Delta t$ is chosen here, our set of schemes is unconditionally stable with no restriction on either space mesh or time increment. For testing purpose only, both computations are performed for $0 \leq t \leq 1$. When $t>>1, U(x, t) \rightarrow 0$.

The first example is as described below:

$$
\left\{\begin{array}{l}
U_{t}=U_{x x}, \quad 0<x<1, \quad t>0, \\
U(x, 0)=\cos (\pi x), \quad U_{x}(0, t)=0, \quad U_{x}(1, t)=0,
\end{array}\right.
$$




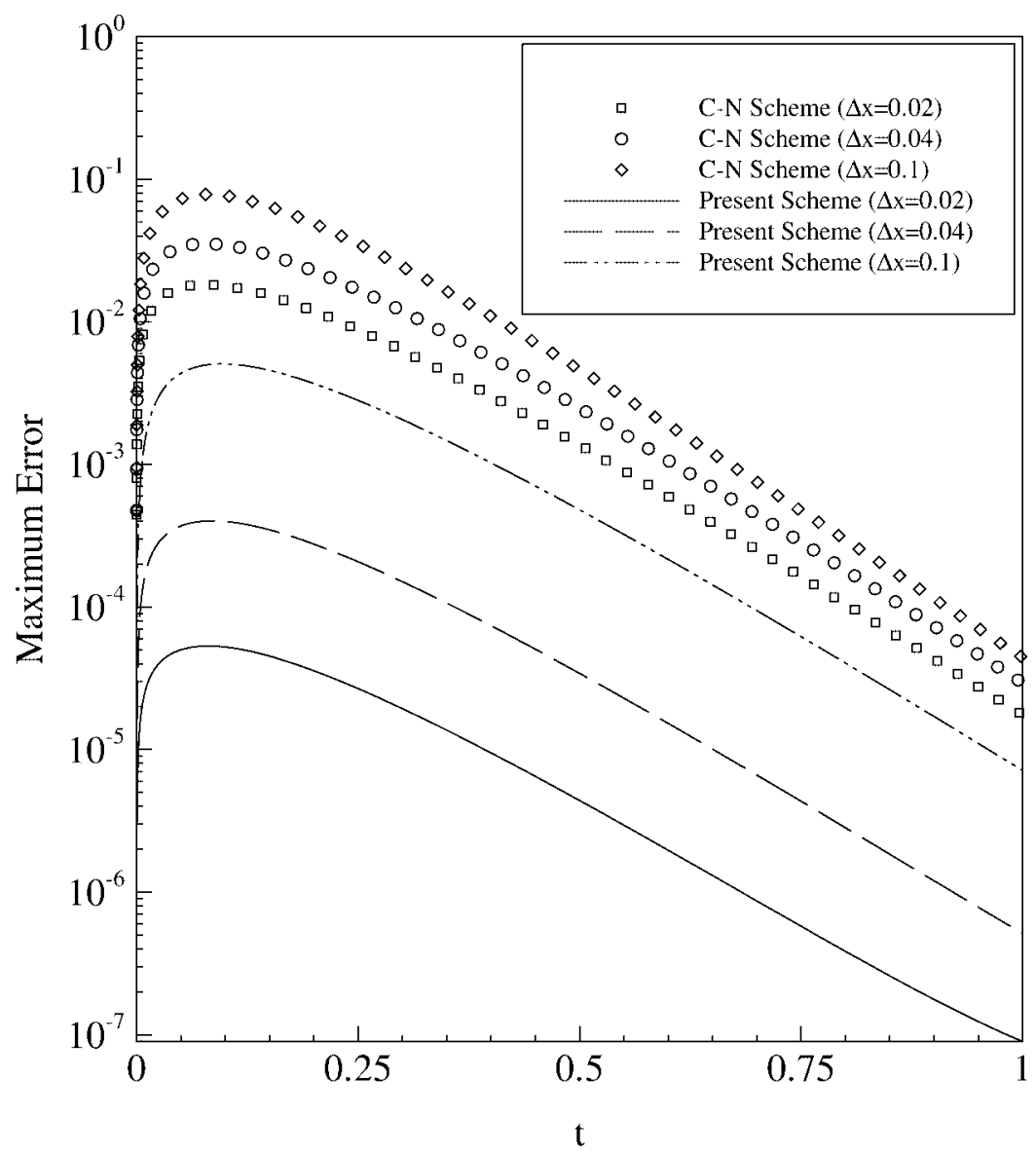

FIG. 1. Comparison results for Example 1.

where the exact solution is $U(x, t)=e^{-\pi^{2} t} \cos (\pi x)$. The homogeneous Neumann boundary conditions indicate that the boundaries are insulated. The comparison results are displayed in Fig. 1. It can be seen from Fig. 1 that the accuracy of the new set of schemes is significantly better than that of the Crank-Nicolson type of scheme.

The second example is:

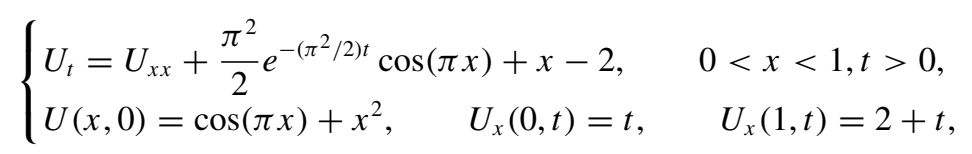

where the exact solution is $U(x, t)=e^{-\left(\pi^{2} / 2\right) t} \cos (\pi x)+x^{2}+x t$. The nonhomogeneous boundary conditions indicate that heat exchanges with the outside through the boundaries. The comparison results are displayed in Fig. 2. The figure once again confirms the fact that the new set of schemes is significantly more accurate than the Crank-Nicolson type of scheme. 


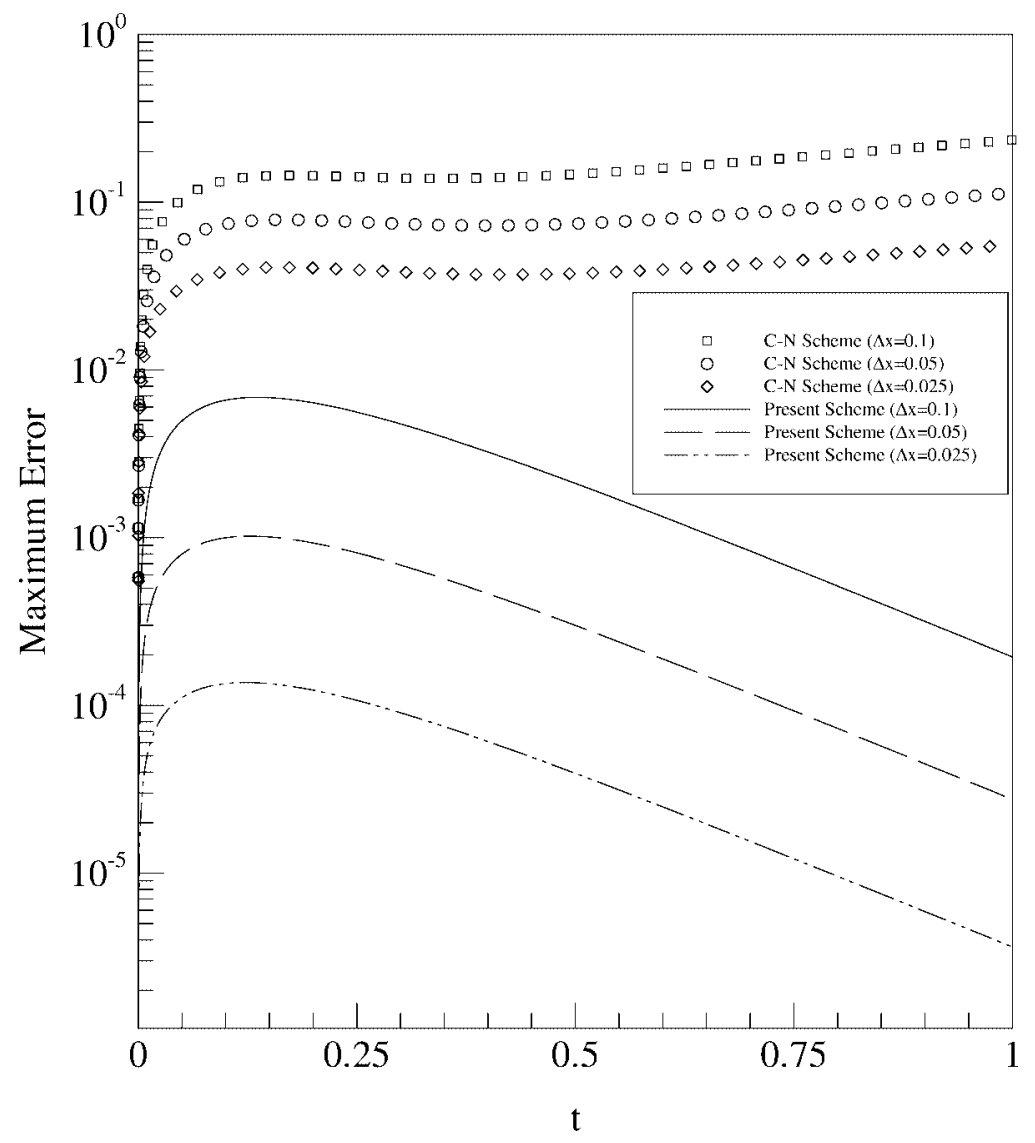

FIG. 2. Comparison results for Example 2.

\section{CONCLUSIONS}

In this article, a set of fourth-order compact difference schemes is constructed for a heat conduction problem with Neumann boundary conditions. The greatest significance of this set of schemes, comparing to other similar ones, is that it is fourth order accurate in space at all grid points, including both interior and boundary points. This set of schemes is also proved to be globally solvable and unconditionally stable. Numerical examples are provided to confirm the accuracy.

\section{References}

1. M. H. Carpenter, D. Gottlieb, and S. Abarbanel, Stable and accurate boundary treatments for compact, high-order finite difference schemes, Appl Numer Math 12 (1993), 55-87.

2. I. Christie, Upwind compact finite difference schemes, J Comput Phys 53 (1985), 353-368.

3. P. C. Chu and C. Fan, A three-point combined compact difference scheme, J Comput Phys 140 (1998), 370-399.

4. S. K. Lele, Compact finite difference schemes with spectral-like resolution, J Comput Phys 103 (1992), $16-42$. 
5. G. F. Carey and W. F. Spotz, High-order compact mixed methods, Commun Numer Methods Eng 13 (1997), 553-564.

6. P. C. Chu and C. Fan, A three-point six-order nonumniform compbined compact difference scheme, J Comput Phys 148 (1999), 663-674.

7. W. Dai and R. Nassar, A compact finite difference scheme for solving a three-dimensional heat transport equation in a thin film, Numer Methods Partial Differential Eq 16 (2000), 441-458.

8. W. Dai and R. Nassar, Compact ADI method for sloving parabolic differential equations, Numerical Methods Partial Differential Eq 18 (2002), 129-142.

9. X. Deng and H. Maekawa, Compact high-order accurate nonlinear scheme, J Comput Phys 130 (1997), $77-91$.

10. D. Gaitonde and J. S. Shang, Optimized compact-difference-based finite volume schemes for linear wave phenomena, J Comput Phys 138 (1997), 617-643.

11. L. Ge and J. Zhang, High accuracy iterative solution of convection diffusion equation with boundary layer on nonuniform grids, J Comput Phys 171 (2001), 560-578.

12. H. Sun, J. Zhang, and J. Zhao, High order compact scheme with multigrid local mesh refinement procedure for convection diffusion problems, Comp Meth Appl Mech and Eng 191(41-42) (2002), 4661-4674.

13. K. E. Atkinson, An introduction to numerical analysis, 2nd ed., John Wiley \& Sons, New York, 1988.

14. J. C. Strikwerda, Finite Difference Schemes and Partial Differential Equations, Chapman \& Hall, New York, 1989. 